\title{
Aplicación del Punto de Umbral en la Educación no Presencial y la Educación Convencional, en el caso de la Carrera de Licenciado Agropecuario
}

Application of the Threshold Point in Non-Presential Education and Conventional Education, in the case of Bachelor of Science in Agriculture

Aplicação limiar Point em não Presencial Educação convencional e Educação, no caso de Agrícola graduada da carreira

DOI: http://dx.doi.org/10.23913/ride.v7i14.273

Claudio Rafael Vásquez Martínez

Universidad de Guadalajara, México crvasquezm@gmail.com

Felipe Anastacio González González Universidad Autónoma de Tamaulipas, México felgonzale@docentes.uat.edu.mx

Erik Moisés Betancourt Núñez

Universidad Autónoma de Tamaulipas, México ebetan@docentes.uat.edu.mx

Sergio Esteban Rodríguez Ramírez Universidad Autónoma de Tamaulipas, México sergiordz-13@outlook.com

Joaquín Torres Mata

Universidad Autónoma de Tamaulipas, México jtorresma@uat.edu.mx 


\section{Resumen}

Las Instituciones de Educación Superior, tanto públicas como privadas, procuran llevar a cabo una política universitaria que trata de remediar dificultades académicas, administrativas, docentes y económicas, rechazan el procedimiento de aquellas condiciones que explican tales adversidades. En el mejor de los casos, se observan dificultades demasiados concretas, como la falta tradicional de elementos y equipo, la escasa formación del personal de ciertos niveles administrativos, la incongruencia de locales, elaboración de módulos, presentación de asesorías, y obviamente se establece una relación directa entre la magnitud de estas dificultades y la carencia de recursos financieros. Este estudio aplicó la técnica "Punto de Umbral", de los Valores en Educación No Presencial y Educación Convencional en Instituciones de Educación Superior. El estudio presenta la relación de Valores Fijos y Valores Variables además de la influencia que tiene el número de alumnos en el Valor Total Semestral por Programa, en el caso concreto de la Carrera de Licenciado Agropecuario. Concluyendo que la educación no presencial es menos costosa que la educación convencional.

Palabras clave: Técnicas comparativas, Educación presencial, Educacion no presencial, Punto de Umbral.

\section{Abstract}

Institutions of Higher Education, both public and private, seek to carry out a University policy that tries to remedy difficulties academic, administrative, educational and economic, reject the procedure of those conditions that explain such adversities. In the best of cases, difficulties can be seen too many concrete, as the traditional lack of elements and equipment, poor training of staff of certain administrative levels, the incongruity of premises, preparation of modules, presentation of consultants, and obviously establishes a direct relationship between the magnitude of these problems and the lack of financial resources. This study applied the technique "Threshold Point" of values in Distance Education and Conventional Education in Institutions of Higher Education. The study presents the relationship of Fixed and Variable Values as well as the influence that has the number of students in the half-yearly total by program, in the case of the Bachelor of 
Science in Agriculture. Concluding that the Distance Education is less expensive than Conventional Education.

Key words: Comparative techniques, Face-to-face education, Distance education, Threshold point.

\section{Resumo}

Instituições de Ensino Superior, públicas e privadas, visam a prossecução de uma política universitário que tenta remediar dificuldades acadêmicas, administrativas, educacionais e econômicas, rejeitar as condições de processo que explicam tais adversidades. No melhor dos casos, muitas dificuldades específicas, como a tradicional falta de elementos e equipamentos, má formação de determinados níveis administrativos, a incongruência de edifícios, o desenvolvimento de módulos, apresentação de conselho são observadas e, obviamente, estabelece um relação directa entre a magnitude dessas dificuldades e da falta de recursos financeiros. Este estudo aplicou a técnica de "ponto crítico" de valores em distnace Educação e Educação convencional em Instituições de Ensino Superior. O estudo apresenta os Securities relação fixa e valores variáveis, além da influência do número de alunos no valor Semestre Programa total no caso de Pós-Graduação Agrícola Race. Concluindo que a educação não-face é menos onerosa do que a educação convencional.

Palavras-chave: técnicas comparativas, educação em sala de aula, Educação distnace, ponto crítico.

Fecha Recepción: Julio 2016 Fecha Aceptación: Diciembre 2016 


\section{Antecedentes}

El Instituto Colombiano para el Fomento de la Educación Superior (ICFES, 2006) procesó una metodología para la determinación de los valores universitarios ante la necesidad de tener una herramienta funcional que permitiera adelantar estudios de valor y se constituyera en mecanismo capaz de producir información sistematizada, útil para el procesamiento de teorías y políticas sobre Educación Superior.

En el informe final del estudio Universidad Desescolarizada (Arboleda, 2006), se presentan seis modelos de Valor en Educación no presencial que son: período de recuperación del capital conforme a libros, tasa de rentabilidad interna, contribución al costo actual neto, costo anual equivalente y costo de Scheneider-Sigelen. Todos aplicados teóricamente a Educación No Presencial, sin ninguna adaptación al sistema de valores de la University of Antioch y sin tener en cuenta la Educación Convencional.

\section{Objeto de Estudio}

El objeto de este estudio es la aplicación de la técnica "Punto de Umbral" de los valores en Educación No Presencial (ENP) y Educación Convencional (EC) en la Carrera de Ingeniero Agronomo. El estudio presenta la relación de valores fijos y valores variables, y el poder que tiene el número de alumnos en el Valor Total Semestral en la Carrera de Licenciado Agropecuario, Facultad de Educación, University of Antioch en los periodos A hasta I (1990-2007).

\section{Objetivos}

- Precisar el valor fijo, el valor variable y el Valor Total Semestral de la Carrera de Licenciado Agropecuario.

- A través de la técnica "Punto de Umbral" realizar la comparación de los valores mencionados anteriormente, entre Educación No Presencial (ENP) y Educación Convencional (EC) en el programa de la Carrera de Licenciado Agropecuario. 


\section{Hipótesis}

Los valores en Educación No Presencial (ENP) de la Carrera de Licenciado Agropecuario son menores que en Educación Convencional (EC) de la carrera de Licenciado Agropecuario.

\section{Preguntas de Investigación}

¿Cuál es el valor fijo en Educación No Presencial (ENP) de la Carrera de Licenciado Agropecuario y en Educación Convencional (EC) de la Carrera de Licenciado Agropecuario?

¿Cuál es el valor variable en Educación No Presencial (ENP) de la Carrera de Licenciado Agropecuario y en Educación Convencional (EC) de la Carrera de Licenciado Agropecuario?

¿Cuál es el "Punto de Umbral" en Educación No Presencial (ENP) de la Carrera de Licenciado Agropecuario y en Educación Convencional (EC) de la Carrera de Licenciado Agropecuario?

\section{Justificación}

En las instituciones de educación superior, públicas y privadas, se desconocen muchas políticas financieras para remediar las dificultades académicas, administrativas, docentes y económicas. Se hace necesario estudiar los valores universitarios con sus respectivas condiciones de valores fijos, variables y valores totales, para así diferenciar en cada proceso administrativo lo que se está erogando. En el mejor de los casos, se conocen dificultades demasiados específicas como la falta tradicional de elementos y equipo, la poca formación del personal de ciertos niveles administrativos, la incongruencia de locales, elaboración de módulos, presentación de asesorías y obviamente se establece una relación directa entre la magnitud de estas dificultades y la carencia de recursos financieros. Por tal motivo en éste estudio se enfatiza en la búsqueda del "Punto de Umbral" (PU) en Educación No Presencial 
(ENP) de la Carrera de Licenciado Agropecuario, en Educación Convencional (EC) de la Carrera de Licenciado Agropecuario.

Revisión de Factores Críticos de Valores en Educación No Presencial (ENP) y en Educación Convencional (EC)

Los Valores en Educación No Presencial (ENP) y en Educación Convencional (EC)

\section{Valores Sociales}

Estudios de valores observan que a nivel mundial, han pasado los días en los que la adjudicación de fondos públicos para la educación, tenía la máxima prioridad (Snowden y Daniel, 2009; Black, 2001; Hirt, 2012).

Es en la década de los 90 cuando se hace evidente una mayor preocupación por determinar la adjudicación de recursos en el campo de la educación y cuando los economistas estudian más intensamente los valores educacionales (Krawitz, 2015; Otley, 2014; Streeck, 2005). Se observa al mismo tiempo una disposición a sustituir la fuerza laboral por bienes de capital. La fuerza laboral en la educación tradicional representa el reglón más alto en los valores, alcanzando porcentajes del $80 \%$ y $90 \%$ por concepto de salarios. Y no solo eso, los aumentos que se producen en los valores de la fuerza laboral repercuten en otros valores de la producción (Barr, 2011; Knorr, 2004; White, 2001).

En Educación Convencional (EC), el salario de los profesores representa el mayor componente del total de valores. Por el contrario, se ha determinado que en Educación No Presencial (ENP) los tres factores más importantes a este respecto son: el "Sistema Multimedial", la "Organización Curricular" y el "Número de estudiantes" (Morrill, 2007; Mackinnon, 2014; Levin, 2015). 


\section{Organización Curricular}

Entre más amplia y variada sea la gama de cursos que ofrezca un modelo de Educación No Presencial (ENP), mayores serán los valores que su producción y mantenimiento generen (Shah, 2005; Tarba, 2017; Frees, 2014).

\section{Número de Estudiantes}

A propósito de lo que ocurre en la Educación Convencional (EC), en la que un mayor número de estudiantes implica necesariamente la contratación de más profesores, en la Educación No Presencial (ENP) esta variable es la que permite que, una vez alcanzado un punto crítico, el incremento que se produzca en el número de estudiantes hace que el valor promedio por estudiante sea cada vez más bajo (Sulej, 2015; Bahiigwa, 2001; Knorr y Bruegger, 2002).

Recapitulando, la Educación No Presencial (ENP) es viable económicamente en pequeña escala, siempre y cuando los medios que se utilicen estén acordes con el tamaño de la población que atienden y sean adecuados desde el punto de vista de los valores. Estos a su vez, en Educación No Presencial (ENP), están más sujetos a las variaciones producidas por cambios en las políticas institucionales (Mirowski, 2002; Gill, 2005; Shah, 1978). Esto implica que el éxito económico de los modelos en Educación No Presencial (ENP) pequeños, dependan en mucho de la inteligencia que se utilice en el designación de políticas y de una administración experimental (Azad, 2007; Gill y Gill, 2009; Tilak, 2006).

Valores fijos y Valores variables en Educación No Presencial (ENP) y en Educación Convencional (EC)

Los valores fijos en un sistema educativo son aquellos que se dan independientemente del número de estudiantes matriculados. Por el contrario, los valores variables son los que dependen directamente de la matrícula (Burker y Serban, 2008; Ghosh, 2007; Glezerman, 2016). 
En Educación Convencional (EC), los valores variables son normalmente más altos que los valores fijos, ya que como se ha dicho; los salarios de los profesores representan la variable más importante en cuanto a valores (Brown, 2015; Shah, 1983; Knorr, 1981).

Los valores fijos más importantes en los que incurre la Educación No Presencial (ENP) son: producción de materiales, transmisión de programas y administración (Barr, 2011; Shah, 1984; Ansari, 1997).

\section{Valores individuales en Educación No Presencial (ENP)}

Entendiéndose por éste, el valor total que paga un estudiante durante un semestre para su sostenimiento en el programa o carrera que está cursando. Entre tales variables están: transporte, alimentación, alojamiento, materiales impresos y módulos, útiles y papelería (Kulakowski, 2006; Ghosh, 1983; Smith y Snowden, 1983).

\section{Categorías Conceptuales}

VALOR: Las erogaciones de mantenimiento para el funcionamiento de un programa, licenciatura u organización, sin tener en cuenta el costo individual y también sin tener en cuenta los ingresos de que se privan los estudiantes al estar estudiando y no laborando (Levin, 2015, Jongbloed, 2000, Hirt, 2012).

VALOR TOTAL SEMESTRAL POR PROGRAMA: Es la erogación de mantenimiento total semestral para el funcionamiento de la Licenciatura en su modalidad respectiva, comprendiendo sus erogaciones de servicios personales, erogaciones generales, erogaciones financieras, arrendamientos, depreciaciones, amortizaciones, además las erogaciones correspondientes de las unidades administrativas centrales (prorrateo), unidades académicas (prorrateo) y unidades de otras dependencias con sus programas especiales (prorrateo). (Nota: Los prorrateos fueron dados con base en el porcentaje de alumnos). Esto es dado de acuerdo a los "criterios" de la Oficina de Planeación de la University of Antioch y son acordados entre ésta y los directivos de la Facultad de Educación, en una entidad gubernamental de la República de Colombia. 
El total se multiplica por el índice inflacionario y devaluativo semestral, tomando como deflactor con respecto al periodo A.

NOTA: El índice inflacionario y devaluativo semestral desde $\mathrm{A}$ hasta $\mathrm{H}$ se tomó como deflactor con respecto al periodo A en cada uno de los valores del presente estudio, suministrado por el Departamento Administrativo Nacional de Estadística (DANE), Bogotá. Mostrado en la siguiente tabla:

Tabla 1. Indice inflacionario y devaluativo semestral desde A hasta $\mathrm{H}$ con respecto al periodo A

\begin{tabular}{|c|c|}
\hline $\begin{array}{c}\text { INDICE INFLACIONARIO } \\
\text { Y DEVALUATIVO }\end{array}$ & PERIODO SEMESTRE \\
\hline 0.83420 & $\mathrm{~A}$ \\
\hline 0.76418 & $\mathrm{~B}$ \\
\hline 0.65270 & $\mathrm{C}$ \\
\hline 0.59890 & $\mathrm{D}$ \\
\hline 0.53140 & $\mathrm{E}$ \\
\hline 0.50580 & $\mathrm{~F}$ \\
\hline 0.45590 & $\mathrm{G}$ \\
\hline 0.43669 & $\mathrm{H}$ \\
\hline
\end{tabular}

Fuente: (según DANE).

SEMESTRE ACADEMICO: Es el número de semanas académicas que dura uno de los periodos en que suele dividirse un programa académico, generalmente separado de otro por un período de vacaciones; para efectos de medir su duración en semanas académicas se tiene en cuenta el primer día de clases hasta el día del último examen.

En el presente estudio el semestre académico se considera de 16 semanas.

NUMERO DE ALUMNOS DEL PROGRAMA: Es el número de alumnos matriculados en un determinado programa, independientemente del número de asignaturas que toman, pero ciñéndose a las reglamentaciones específicas de la University of Antioch. La información se toma aproximadamente tres meses después del día de iniciación de clases, cuando se supone que termina el período de adiciones y cancelaciones de asignaturas y matrículas. 
EDUCACION CONVENCIONAL (EC): Sistema o modalidad de educación que propicia el proceso enseñanza-aprendizaje, básicamente en forma presencial.

EDUCACIÓN NO PRESENCIAL (ENP): Sistema o modalidad de educación que se apoya en la utilización de multimedios que propician el proceso enseñanza-aprendizaje básicamente en forma no presencial, con el fin de lograr objetivos educativos con una cobertura geográfica y demográfica mayor que la de un sistema de Educación Convencional.

VALOR TOTAL: Es la adición del Valor Fijo y el Valor Variable en cada programa, Licenciatura.

"PUNTO DE UMBRAL": Para el presente estudio el punto de umbral es la intersección de las gráficas de valor total en Educación Convencional (EC) y costo total en Educación No Presencial (ENP) correspondiente a cada programa Licenciatura, y dicho punto indica que en ese espacio los valores totales son iguales en Educación Convencional (EC) y Educación No Presencial (ENP).

Limitaciones: El presente estudio, se limita por su confidencialidad en los datos y las anormalidades académicas que ha tenido la University of Antioch. Los valores fijos y los valores variables, serán determinados en forma indirecta, por lo que su valor será aproximado. El costo se limita a las erogaciones de mantenimiento para el funcionamiento de un programa u organización, sin tener en cuenta el costo individual y también sin tener en cuenta los ingresos de que se privan los alumnos al estar estudiando y no laborando.

\section{Metodología}

El presente estudio se basa en los valores universitarios de la obra "La Educación a Distancia en Antioquia de la Teoría a la Realidad” (Vásquez y Restrepo, 2005).

\section{Población}

La población para el análisis de valores educacionales en Educación Convencional (EC) y en Educación No Presencial (ENP) en la University of Antioch, es la siguiente: 
En Educación Convencional (EC), Pregrado, se tendrán en cuenta el siguiente programa, Licenciado en Agropecuaria.

En Educación No Presencial (ENP), Pregrado, se tendrán en el siguiente programa, Licenciado en Agropecuaria.

\section{Variables}

\section{Valor Total Semestral por Programa}

Licenciado Agropecuario (EC) 211624.

Tabla 2. Periodo - número alumnos - valor total semestral por Carrera de Licenciado Agropecuario - EC en pesos constantes con respecto al periodo A.

\begin{tabular}{|c|c|c|}
\hline PERIODO SEMESTRE & NUMERO DE ALUMNOS & $\begin{array}{c}\text { COSTO TOTAL SEMESTRAL } \\
\text { POR PROGRAMA,CARRERA } \\
\text { LICENCIADO } \\
\text { AGROPECUARIO (EC) }\end{array}$ \\
\hline A & 2 & 211,624 \\
\hline B & 2 & 223,783 \\
\hline C & 17 & 449,367 \\
\hline D & 14 & 344,834 \\
\hline E & 14 & 412,812 \\
\hline F & 13 & 329,809 \\
\hline G & 15 & 377,625 \\
\hline H & 15 & 371,138 \\
\hline I & 15 & 396,405 \\
\hline
\end{tabular}

\section{Valor Total Semestral por Programa}

Licenciado Agropecuario (ENP) 397834.

Para los otros semestres se aplicó semejante procedimiento trabajando de antemano con pesos constantes con respecto al periodo A realizándose así la siguiente tabla: 
Tabla 3. Periodo - número alumnos - valor total semestral por programa licenciatura - ENP. En pesos constantes con respecto al periodo A.

\begin{tabular}{|c|c|c|}
\hline PERIODO SEMESTRE & NUMERO DE ALUMNOS & $\begin{array}{c}\text { COSTO TOTAL SEMESTRAL } \\
\text { POR PROGRAMA,CARRERA } \\
\text { LICENCIADO } \\
\text { AGROPECUARIO (EC) }\end{array}$ \\
\hline A & 2 & 397,834 \\
\hline B & 2 & 404,342 \\
\hline C & 17 & 457,285 \\
\hline D & 14 & 490,927 \\
\hline E & 14 & 496,385 \\
\hline F & 13 & 442,772 \\
\hline G & 15 & 433,964 \\
\hline H & 15 & 421,376 \\
\hline I & 15 & 426,728 \\
\hline
\end{tabular}

\section{Procedimiento}

Para establecer los valores de cada programa-licenciatura, se consultó la obra "La Educación a Distancia en Antioquia De la Teoría a la Realidad" (Vásquez y Restrepo, 2005) en la que se mencionan los valores totales por semestre para cada programa, así como el número de alumnos de cada programa, esto para cada periodo-semestre desde A hasta I.

Estos valores se corrigieron con los índices inflacionarios y devaluativos mencionados anteriormente. En cada semestre se conoce el número de alumnos para cada programa, de modo que se tienen puntos de la función $\mathrm{VT}=\mathrm{VF}+\mathrm{vD}$, donde:

VT $=$ Valor Total Semestral por Programa

$\mathrm{VF}=$ Valor Fijo.

$\mathrm{v}=$ Valor Marginal (pendiente)

$\mathrm{D}=$ Número de Alumnos 
Tabla 4. Evolución histórica del número de alumnos en programas de EC- University of Antioch, Periodo-Semestre A hasta I.

\begin{tabular}{|c|c|c|c|c|c|c|c|c|c|}
\hline $\begin{array}{c}\text { Periodo Semestre } \\
\text { Programa Licenciado Agropecuario }\end{array}$ & A & B & C & D & E & F & G & H & I \\
\hline & 2 & 2 & 1 & 1 & 1 & 1 & 1 & 1 & 1 \\
& & & 7 & 4 & 4 & 3 & 5 & 5 & 5 \\
\hline
\end{tabular}

Tabla 5. Evolución histórica del número de alumnos en programas de ENP - University of Antioch, Periodo-Semestre A hasta I.

\begin{tabular}{|c|c|c|c|c|c|c|c|c|c|}
\hline $\begin{array}{c}\text { Periodo Semestre } \\
\text { Programa Licenciado Agropecuario }\end{array}$ & A & B & C & D & E & F & G & H & I \\
\hline & 2 & 2 & 6 & 3 & 3 & 5 & 4 & 3 & 3 \\
& 2 & 6 & 3 & 1 & 1 & 3 & 6 & 2 & 2 \\
\hline
\end{tabular}

Gráfica 1. Valor Total Semestral por Programa Licenciado Agropecuario.

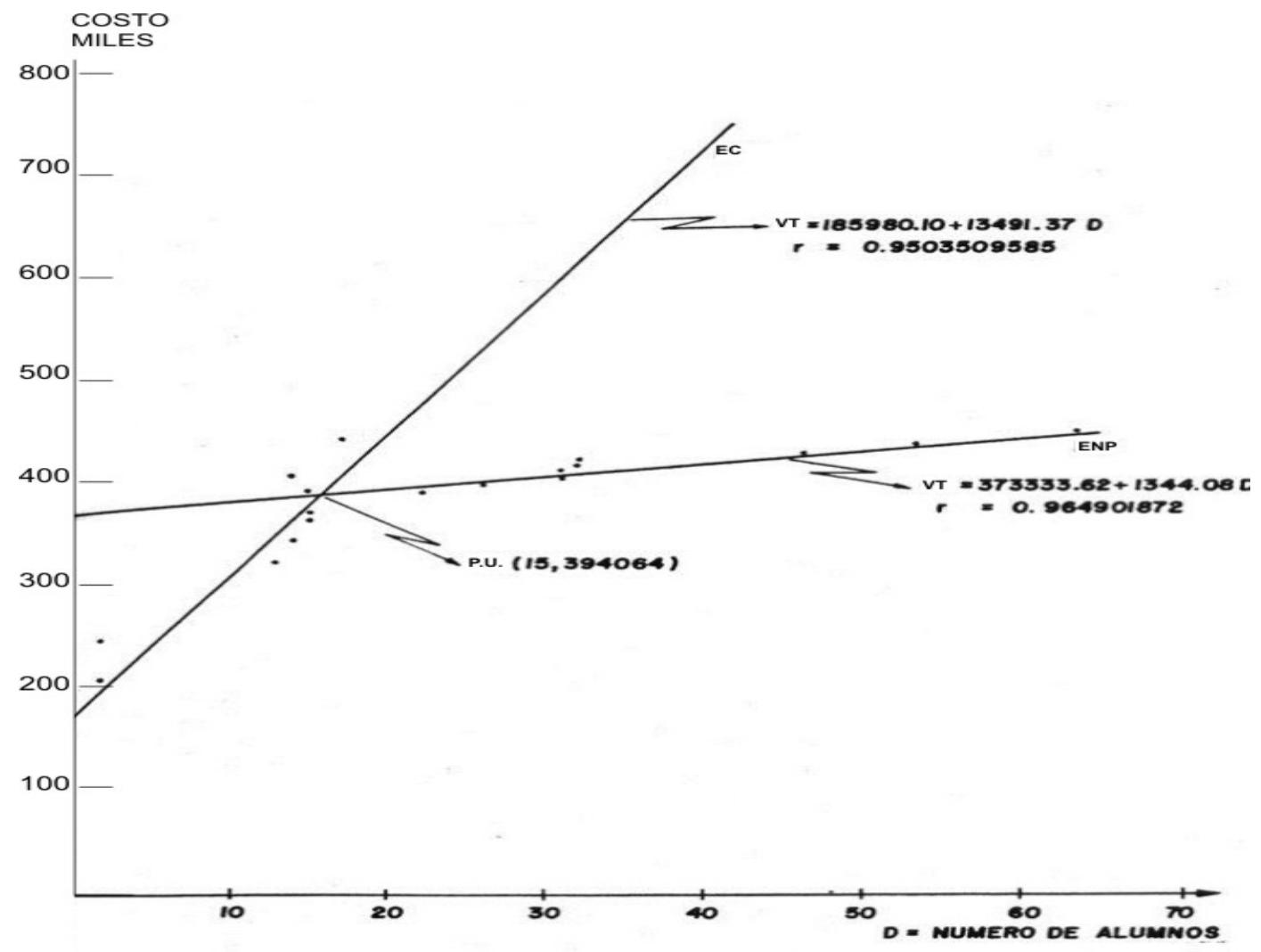


Esto permitió realizar un análisis de cada programa puntualizando la ecuación del Valor Total y el Punto de Umbral.

La información encontrada se condensó en las siguientes tablas:

Tabla 6. Modalidad-ecuación-coeficiente de correlación-punto de umbral en el programa Licenciado Agropecuario en pesos constantes con respecto al periodo-semestre.

\begin{tabular}{|c|c|c|c|}
\hline MODALIDAD & ECUACIÓN & $\begin{array}{c}\text { COEFICIENTE DE } \\
\text { CORRELACIÓN }\end{array}$ & $\begin{array}{c}\text { PUNTO DE } \\
\text { UMBRAL }\end{array}$ \\
\cline { 1 - 3 } $\mathrm{EC}$ & $\mathrm{VT}=185980,10+13491,37 \mathrm{D}$ & 0.9503509585 & $\begin{array}{c}\mathrm{D}=15 \text { Alumnos } \\
\mathrm{VT}=394064 \text { pesos }\end{array}$ \\
\cline { 1 - 3 } $\mathrm{ENP}$ & $\mathrm{VT}=373333,62+1344,08 \mathrm{D}$ & 0.964901872 & \\
\hline
\end{tabular}

Tabla 7. Valor fijo y valor variable con respecto al punto de umbral en pesos constantes del periodo-semestre, A en programas EC - University of Antioch

\begin{tabular}{|l|l|l|}
\hline PROGRAMA & VALOR FIJO & VALOR VARIABLE \\
\hline Agropecuaria & 185980 & 208084 \\
\hline Biología-Química & $4^{\prime} 429507$ & $6^{\prime} 782350$ \\
\hline Docencia Comercial & 288299 & 122995 \\
\hline Electrotecnia & 198051 & 247275 \\
\hline Idiomas Español Literatura & $3^{\prime} 318743$ & $3^{\prime} 534784$ \\
\hline Sociales Geografía Historia & $2^{\prime} 354442$ & $3^{\prime} 186191$ \\
\hline Matemática-Física & $1^{\prime} 382263$ & $1^{\prime} 382263$ \\
\hline Mecánica Industrial & 482437 & 612238 \\
\hline
\end{tabular}


Tabla 8. Valor fijo y valor variable con respecto al punto de umbral en pesos constantes del periodo-semestre A, En programas ENP - University of Antioch

\begin{tabular}{|l|l|l|}
\hline PROGRAMA & VALOR FIJO & VALOR VARIABLE \\
\hline Agropecuaria & 373334 & 20730 \\
\hline Biología-Química & $10^{\prime} 161843$ & $1^{\prime} 050004$ \\
\hline Docencia Comercial & 324659 & 86634 \\
\hline Electrotecnia & 310996 & 134329 \\
\hline Idiomas Español Literatura & $6^{\prime} 432987$ & 420539 \\
\hline Sociales Geografía Historia & $5^{\prime} 190794$ & 349838 \\
\hline Matemática-Física & $3^{\prime} 724327$ & 102906 \\
\hline Mecánica Industrial & 889095 & 205580 \\
\hline
\end{tabular}

\section{Análisis}

En el lapso de tiempo Periodo-Semestre desde A hasta I en la University of Antioch, en las modalidades EC y ENP, referente a valores se presenta el siguiente análisis.

En la gráfica 1 se presentan las ecuaciones de regresión lineal de ENP y EC de la Carrera de Licenciado Agropecuario con su Punto de Umbral (PU).

En Educación Convencional $(\mathrm{EC})$ la regresión lineal es VT $=185980,10+13491,37 \mathrm{D}$ (Tabla 6) con un coeficiente de correlación $r_{1}=0.9503509585$ significativo con $\alpha=0.05$, $n$ 2 g. L., por cuanto el valor teórico en Tabla estadística (Glass y Stanley, 2006) de r = 0.666, g. L. $n-2$ al $\gamma=95 \%$ bilateral, es menor al valor encontrado $r_{1}=0.9503509585$ ( $\left.p<0.01\right)$.

Un Valor Fijo (V. F.) 185980,10 (Tabla 7)

Un Valor Marginal $v_{1}=13491,37$ que es la pendiente de la regresión lineal.

Donde $\mathrm{v}_{1}$ representa el valor necesario por un alumno más en el eje D.

Por equivalencia en Regresión Bivariante la prueba de la pendiente y de correlación son equivalentes.

El coeficiente de correlación $r_{1}=0.9503509585$, indica una buena relación lineal directa positiva entre Y (VT = Valor Total Semestral por Programa $)$ y X (D = Número de alumnos, 
(Tabla 4). Con un coeficiente de determinación $\mathrm{r}^{2}{ }_{1}=90.32 \%$, expresa la proporción de variación total en los valores de la variable Y (VT = Valor Total Semestral por Programa) que puede ser considerada o explicada por una relación lineal con los valores de la variable $\mathrm{X}(\mathrm{D}=$ Número de Alumnos).

En Educación No Presencial (ENP) la regresión lineal es VT $=373333,62+1344,08$ D (Tabla 6) con un coeficiente de correlación $\mathrm{r}^{2}=0.964901872$ significativo con $\alpha=0.05, \mathrm{n}-2$ g. L. por cuanto el valor teórico en Tabla estadística (Glass y Stanley, 2006) de $\mathrm{r}=0.666$, g. L. N-2 al $\gamma=95 \%$ bilateral, es menor al valor encontrado $r_{2}=0.964901872(p<0.01)$.

Un Valor Fijo (C.F.) = 373333,62 (Tabla 8)

Un Valor Marginal $\mathrm{v}_{2}=1344,08$ que es la pendiente de regresión lineal.

Donde $\mathrm{v}_{2}$ representa el valor necesario por un alumno más en el eje D.

Por equivalencia en Regresión Bivariante la prueba de la pendiente y de correlación son equivalentes.

El coeficiente de correlación $\mathrm{r}_{2}=0.964901872$, indica una buena relación lineal, directa positiva entre Y (VT= Valor Total Semestral por Programa) y X (D = Número de alumnos (Tabla 5). Con un coeficiente de determinación $\mathrm{r}_{2}^{2}=93.10 \%$, expresa la proporción de variación total en los valores de la variable Y (VT = Valor Total Semestral por Programa) que puede ser considerada o explicada por una relación lineal con los valores de la variable $\mathrm{X}(\mathrm{D}=$ Número de alumnos).

Cuando la regresión lineal de Educación Convencional (EC) se intersecta con la regresión lineal de Educación No Presencial (ENP), forma el Punto de Umbral (PU), donde en este punto se igualan VT y el número de alumnos (D Tabla 4 y 5) en Educación Convencional (EC) y Educación No Presencial (ENP).

Regresión Lineal en Educación Convencional (EC) : VT = 185980,10 + 13491,37 D Ec.(1)

Regresión Lineal en Educación No Presencial (ENP) : VT = 373333,62 + 1344,08 D Ec.(2)

Igualando VT en ecuaciones (1) y (2) 
$185980,10+13491,37 \mathrm{D}=373333,62+1344,08 \mathrm{D}$

$13491,37 \mathrm{D}-1344,08 \mathrm{D}=373.333,62-185980,10$

$12147,29 \mathrm{D}=187353,52$

$\mathrm{D}=187353,52 / 12147,29=15,42 \approx 15$

En el Punto de Umbral (PU), le corresponde un VT = 394064 pesos constantes con respecto al periodo A, un D = 15 Alumnos (Tabla 10), un Valor Variable (VV) (Tabla 7) en Educación Convencional (EC) de 208084 pesos constantes con respecto al periodo A, un Valor Variable (VV) (Tabla 8) en Educación No Presencial (ENP) de 20730 pesos constantes con respecto al periodo A.

Concluyéndose, si el número de alumnos es mayor de 15 en la Carrera de Licenciado Agropecuario de Educación No Presencial (ENP), es menos costosa que la de Educación Convencional (EC).

\section{Conclusiones}

El Punto de Umbral (PU), tiene validez para la serie de condiciones que existieron durante el período de A a I en el que se emplearon los datos facilitados por la Oficina de Planeación de la University of Antioch.

El Punto de Umbral (PU) tiene en cuenta la experiencia pasada y determina probables efectos que puede producir la proyección del pasado en las operaciones de un futuro inmediato para la University of Antioch, por ejemplo, aumento o disminución de planta física, docentes, materiales didácticos, equipos, reemplazo de planta física y equipos obsoletos.

En los programas de Educación Convencional (EC), las regresiones lineales fueron de la forma VT $=(\mathrm{VF})_{1}+\mathrm{v}_{1} \mathrm{D}$, donde VT es el Valor Total Semestral por Programa, D es el número de alumnos, $(\mathrm{VF})_{1}$ es el termino independiente e indicando un valor fijo, $\mathrm{v}_{1}$ es el valor marginal (pendiente). 
Referente a los coeficientes de correlación (mayores de 0.9) se hallaron significativos con $\alpha$ $=0.05, \mathrm{n}-2$ g.L., por cuanto el valor teórico en tablas estadísticas (Glass y Stanley, 2006) de $r=0.666$, g.L. $n-2$ al $r=95 \%$ bilateral, es menor a los encontrados ( $p<0.01)$,

Por equivalencia en Regresión Bivariante las pruebas de las pendientes y de correlaciones son equivalentes.

Los coeficientes de correlación encontrados en los diferentes programas de Educación Convencional (EC) son buenos (mayores de 0.9), indicando una buena relación lineal directa positiva entre VT y D, de modo que los valores de VF (Valores Fijos en EC) y VV (Valores Variables en EC) deben ser significativos. Y unos coeficientes de determinación también buenos (mayores de 0.8), donde es probable que con esos coeficientes de determinación expresen la proporción de la variación total en los valores de la variable VT que pueden ser consideradas o explicadas por una relación lineal con los valores de la variable D.

En los programas de Educación No Presencial (ENP), las regresiones lineales fueron de la forma $\mathrm{VT}=(\mathrm{VF})_{2}+\mathrm{v}_{2} \mathrm{D}$, donde $\mathrm{VT}$ es el

Referente a los coeficientes de correlación (mayores de 0.9) se hallaron significativos con $\alpha$ $=0.05, \mathrm{n}-2$ g.L., por cuanto el valor teórico en tablas estadísticas (Glass y Stanley, 2006) de $\mathrm{r}=0.666, \mathrm{gL} . \mathrm{n}-2$ al $\gamma=95 \%$ bilateral, es menor a los encontrados. $(\mathrm{p}<0.01)$.

Por equivalencia en Regresión Bivariante las pruebas de las pendientes y de correlaciones son equivalentes.

Los coeficientes de correlación encontrados en los diferentes programas de Educación No Presencial (ENP) son buenos (mayores de 0.9), indicando una buena relación lineal directa positiva entre VT y D, de modo que los valores de VF (Valores fijos en ENP) y VV (Valores variables en ENP) deben ser significativos. Y unos coeficientes de determinación también buenos (mayores de 0.8), donde es probable que con esos coeficientes de determinación expresen la proporción de la variación total en los valores de la variable VT que pueden ser consideradas o explicadas por una relación lineal con los valores de la variable D.

Cuando la regresión lineal del programa respectivo de Educación Convencional (EC) se intersecta con la regresion lineal del programa respectivo de Educación No Presencial 
(ENP), forma el "Punto de Umbral", donde en este punto se iguala VT (Valor Total Semestral por Programa).

Concluyéndose, si el número de alumnos es mayor de la abscisa asignada en el Punto de Umbral en el programa analizado de Educación No Presencial (ENP) entonces tiene menos valor que los de Educación Convencional (EC) en el periodo desde A hasta I. Donde la institución ahorra más recursos de Valor Total Semestral en el programa de Educación No Presencial (ENP) sobre Educación Convencional (EC).

\section{Recomendación}

Se sugiere que para futuros estudios de valores universitarios se analicen con detenimiento los puntos de umbral de cada una de las carreras en cada Institución educativa para obtener los valores variados y los valores fijos de la administración de Instituciones de Educación Superior. 


\section{Bibliografía}

Ansari, M. M. (1997). Cost effectiveness of higher Education: a critical assessment. New Delhi: concept Pub. Co.

Arboleada, T. J. (2006). Informe final del Proyecto de Universidad Desescolarizada. Volúmen IV. Medellín: Edicion Corporación Educativa San Pablo.

Azad, J. L. (2007). Criteria based funding of higher education. Journal of Educational Planning and Administration 12 (4), 25-31.

Bahiigwa, G. (2001). Identification and measurement of indicators of sucess /faire, and sustainability of farming systems in Uganda. Kampala: Economic Policy Research Centre.

Barr, M. J. (2011). Budgets and financial management in higher Education. San Francisco: Jossey-Bass.

Black, J. (2001). Strategic enrollment management revolution. Washington, DC: American Association of Collegiate Registrars and Admissions.

Brown, J. (2015). How the financial crisis and great recession affected higher Education. Chicago: The University of Chicago Press.

Burker, J. C., and Serban. A. M. (2008). Performance funding for public higher education: fad or trends? San Francisco: Jossey-Boss.

Frees, E. (2014). Predictive modeling applications in actuarial science. New York: Cambridge University Press.

Ghosh, D. K. (1983). University system in India. Jabalpur, M.P.: Ragul publications.

Ghosh, D. K. (2007). Financing education; vol. 1-Finance management and planning in education: lessons from the UK system. New Delhi: Cosmo. 
Gill, R. (2005). State, market, and civil society: issues and interface. Jaipur: Rawat Publications.

Gill, T. K., and Gill, S. S. (2009). Financial management of universities in developping countries. Higher Education Policy. 16 (2), 35-36.

Glass, G. V., and Stanley, J. C. (2006). Métodos Estadísticos Aplicados a las Ciencias Sociales. New York: Prentice Hall.

Glezerman, D.R. (2016). Managing \& collecting student receivables. Washington, DC: National Association of College and University Business Officers.

Hirt, G. (2012). Fundamentals of investment management. Dubuque: McGraw-Hill.

ICFES. (2006). Indices para el Análisis de los Valores Universitarios en la Universidad Externado de Colombia. Seminario Nacional sobre Valores Universitarios. Bogotá: ICFES.

Jongbloed, B. W.A. (2000). Spending strategies : a closer look at the financial management of the European University. Geneva: Association of European Universities.

Knorr, K. (1981). Advances in social theory and methodology : toward and integration of micro and macro sociologies. Boston: Routledge \& Kegan Paul.

Knorr, K., and Bruegger, U. (2002). Global gogh microstructures: the virtual societies of financial markets. American Journal of Sociology. 107 (9), 05-51.

Knorr, K., and Preda, A. (2004). The sociology of financial markets. Oxford: Oxford University Press.

Krawitz, N. (2015). The board's role in financial oversight. Washington, DC: AGB Press.

Kulakowski, E.C. (2006). Research administration and management. Sudbury, Mass: Jones and Bartlett.

Levin, G. (2015). Porfolio management: a strategic approach. Boca Ratón: CRC Press. 
Mackinnon, P. (2014). University Leadership and public Policy in the twenty-first Ventura; a President's perspective. Toronto: University of Toronto Press.

Mirowski, P. (2002). Machine Dreams: Economics Becomes a Cyborg Science. Cambridge: Cambridge University Press.

Morril, R. (2007). Strategic Leadership: integrating Strategic and Leadership in colleges and universities. Westport, CT: Praeger.

Otley, D. (2014). Management control and uncertainty. Basingstoke: Palgrave Macmillan.

Shah, K.R. (1978). Optimum utilization of educational expenditure in Gujarat. Bombay: Popular Prakashan.

Shah, K. R. (1983). Adult Education programme in Gujarat. Ahmedabad: Sardar patel Institute of economic and social research.

Shah, K. R. (1984). Education, earnings, and income distribution : an inquiry into equity issues involved in the government financing of higher Education in India: a study of the M.S. University of Barroda. New Delhi: Deep \& Deep.

Shah, K.R. (2005). University-relations in financing higher Education: some issues. Journal of educacional planning and administration. $16(2), 24-26$.

Smith, W. A.S. and Snowden, B. L. (1983). A review of distance Education in Ontario Universities. Toronto: The Council of Ontario Universities.

Snowden, B. L., and Daniel, J. S. (2009). The economics of small open universities. Open University Conference on the Education of Adults at a Distance. 15 (3), 18-23.

Streeck, W. (2005). The sociology of labor markets and trade unions. Princeton: Princeton University Press.

Sulej, J. (2015). Rethinking businesss schools. New York: Palgrave Macmillan. 
Tarba, S. (2017). Mergers and Acquisitions in practice. New York: Routledge.

Tilak, J. B. G. (2006). Student loans as the answer to lack of resources in higher education. Economic and Political Weekly. 34 (2), 2-15.

Vasquez, C. R., y Restrepo, B. (2005). La Educación a Distancia en Antioquia. De la Teoría a la realidad. Bogotá: Ed. Guadalupe.

White, H. (2001). Markets from networks. Princeton: Princeton University Press. 